Mathematical Modelling And Analysis

Volume 16 Number 1, March 2011, 52-61

Doi:10.3846/13926292.2011.559628

(C) Vilnius Gediminas Technical University, 2011
www.tandf.co.uk/journals/TMMA

Publisher: Taylor\&Francis and VGTU

Online ISSN: 1648-3510

Print ISSN: 1392-6292

\title{
On Some Fučík Type Problem with Cubic Nonlinearity
}

\section{N. Sergejeva}

\author{
Department of Mathematics and Natural Sciences \\ Parades 1, LV-5400 Daugavpils, Latvia \\ E-mail: natalijasergejeva@inbox.lv
}

Received September 1, 2010; revised December 14, 2010; published online March 1, 2011

\begin{abstract}
We study the second order Fučík type problem with cubic nonlinearity $-x^{\prime \prime}=\mu\left(x^{+}\right)^{3}-\lambda\left(x^{-}\right)^{3}, x(0)=0, x(1)=\gamma \int_{0}^{1} x(s) d s$ and construct the Fučík spectrum for this problem. The spectrum obtained under normalization condition (otherwise problem may have continuous spectra) structurally is similar to Fučík spectra for the problem $-x^{\prime \prime}=\mu x^{+}-\lambda x^{-}$with the same boundary conditions.
\end{abstract}

Keywords: Fučík equation, Fučík spectrum, integral condition, lemniscatic function.

AMS Subject Classification: 34B15.

\section{Introduction}

There is intensive literature about asymmetric ordinary differential equations. One of the pioneering work is the article by S. Fučík which can be found in the book [4]. In the mentioned source a specific formally nonlinear equation is considered containing two spectral parameters. The classical (and the simplest) equation of this kind is a famous Fučík equation

$$
\begin{aligned}
& -x^{\prime \prime}=\mu x^{+}-\lambda x^{-}, \quad \mu, \lambda \in \mathbb{R}, \\
& x^{+}=\max \{x, 0\}, \quad x^{-}=\max \{-x, 0\} .
\end{aligned}
$$

The Fučík problem is a problem of finding a set of parameters $(\mu, \lambda)$ such that the problem (1.1),

$$
x(0)=0, \quad x(1)=0
$$

has nontrivial solutions. This set is called a Fučík spectrum.

The problem (1.1), (1.2) generalizes classical spectral problems with one parameter and reduces to the classical problem if $\mu=\lambda$. The Fučík spectrum is a two-dimensional set which may have interesting properties. The Fućík spectrum for the problem (1.1), (1.2) is well known and consists of infinite set of curves (branches) $F_{i}^{+}$and $F_{i}^{-}(i=0,1,2, \ldots)$ which can be obtained analytically and graphically. The lower index shows how many zeroes has the 
respective solution in the interval $(0 ; 1)$, and the upper index shows the sign of the derivative of a solution at $t=0$. Some of the branches for the spectrum of the classical Fučík problem are shown in Fig. 1.
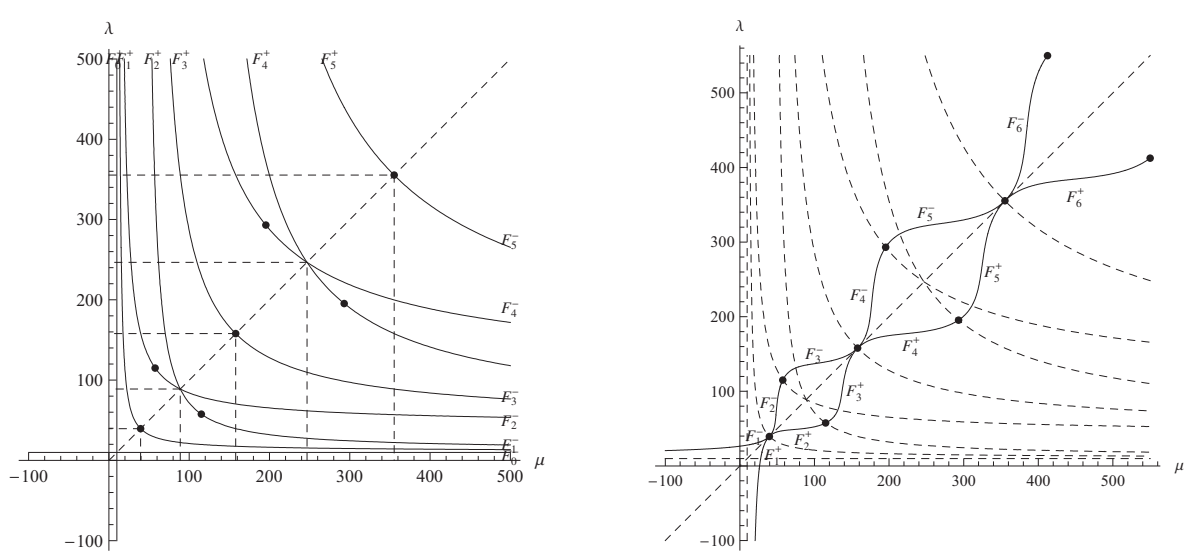

Figure 1. The spectrum for the problems (1.1), (1.2) and (1.1), (1.3).

Since the work of Fučík, this equation has been studied together with different boundary conditions.

There is little information about Fučík type problems with the integral conditions. The investigations for linear differential equations with one parameter and integral conditions were made in the works $[1,5,6]$. Some new investigations of Green's function for second-order problems with nonlocal boundary condition (inc. integral conditions) are presented in papers [7, 13]. There are some author's works devoted to Fučík type problem with integral condition. The results about the Fučík equation (1.1) with zero integral condition

$$
x(0)=0, \quad \int_{0}^{1} x(s) d s=0
$$

were considered in the work [9]. The spectrum of the problem (1.1), (1.3) is essentially different from the classical one. It is not a countable set of hyperbolas, but a two-sided wave spreading along the bisectrix of the first quadrant in the parameter $(\mu, \lambda)$ plane. Some of the branches for the spectrum of the problem (1.1), (1.3) are depicted in Fig. 1.

The results about the Fučík equation (1.1) with conditions

$$
x(0)=0, \quad x(1)=\gamma \int_{0}^{1} x(s) d s, \quad \gamma \in \mathbb{R} .
$$

which include both conditions (1.2) and (1.3) were considered in [12]. The description of the spectrum for different values of $\gamma$ was given too.

In the present work we study the Fučík type problems which include equation with nonlinear (cubic) functions on the right side

$$
-x^{\prime \prime}=\mu\left(x^{+}\right)^{3}-\lambda\left(x^{-}\right)^{3}, \quad \mu \geq 0, \lambda \geq 0
$$

and different boundary conditions. 
The paper is organized as follows. Section 2 is devoted to the spectrum of the Fučík type problem which includes the equation (1.5) and Dirichlet boundary conditions. This spectrum was considered in [8] for different power functions. In Section 3, we present the results about the problem which includes the equation (1.5) and zero integral condition. The description of the spectrum was given in the work [10] (or in the work [11]) where the equation (1.5) was considered in the form

$$
-x^{\prime \prime}=2 \mu^{4}\left(x^{+}\right)^{3}-2 \lambda^{4}\left(x^{-}\right)^{3}, \quad \mu>0, \quad \lambda>0 .
$$

In Section 4, we provide explicit formulas for the spectrum of the problem (1.5), (4.1) and graphically construct the spectrum for different $\gamma$ values. The analysis of the spectrum of this problem for different $\gamma$ values is the main goal of this work. The expressions of the spectrum branches are carried out using the lemniscatic functions. The lemniscatic sine and cosine functions can be expressed in terms of the Jacobi elliptic functions as [14]

$$
\operatorname{sl} t=k \frac{\operatorname{sn}(t / k)}{\operatorname{dn}(t / k)}, \quad \operatorname{cl} t=\operatorname{cn} \frac{t}{k}, \quad k=\frac{1}{\sqrt{2}} .
$$

The formulas for relations between lemniscatic functions and their derivatives are known from [2]. The spectrum is obtained under the normalization condition $\left|x^{\prime}(0)\right|=1$, because otherwise problems may have continuous spectra.

\section{The Spectrum of the Problem with Dirichlet Condi- tions}

Consider the equation (1.5) with the conditions

$$
x(0)=0, \quad x(1)=0, \quad\left|x^{\prime}(0)\right|=1 .
$$

Theorem 1 [see [8]]. The Fučik spectrum for the problem (1.5), (2.1) consists of the branches given by

$$
\begin{aligned}
& F_{0}^{+}=\left\{\left(2(2 A)^{4}, \lambda\right)\right\}, \quad F_{0}^{-}=\left\{\left(\mu, 2(2 A)^{4}\right)\right\}, \\
& F_{2 i-1}^{+}=\left\{(\mu, \lambda) \mid 2 A \sqrt[4]{\frac{2}{\mu}} i+2 A \sqrt[4]{\frac{2}{\lambda}} i=1\right\}, \\
& F_{2 i}^{+}=\left\{(\mu, \lambda) \mid 2 A \sqrt[4]{\frac{2}{\mu}}(i+1)+2 A \sqrt[4]{\frac{2}{\lambda}} i=1\right\}, \\
& F_{i}^{-}=\left\{(\mu, \lambda) \mid(\lambda, \mu) \in F_{i}^{+}\right\}
\end{aligned}
$$

where $A=\int_{0}^{1} \frac{d s}{\sqrt{1-s^{4}}}, i=1,2, \ldots$

The first five pairs of branches of the spectrum to the problem (1.5), (2.1) are depicted in Fig. 2. 

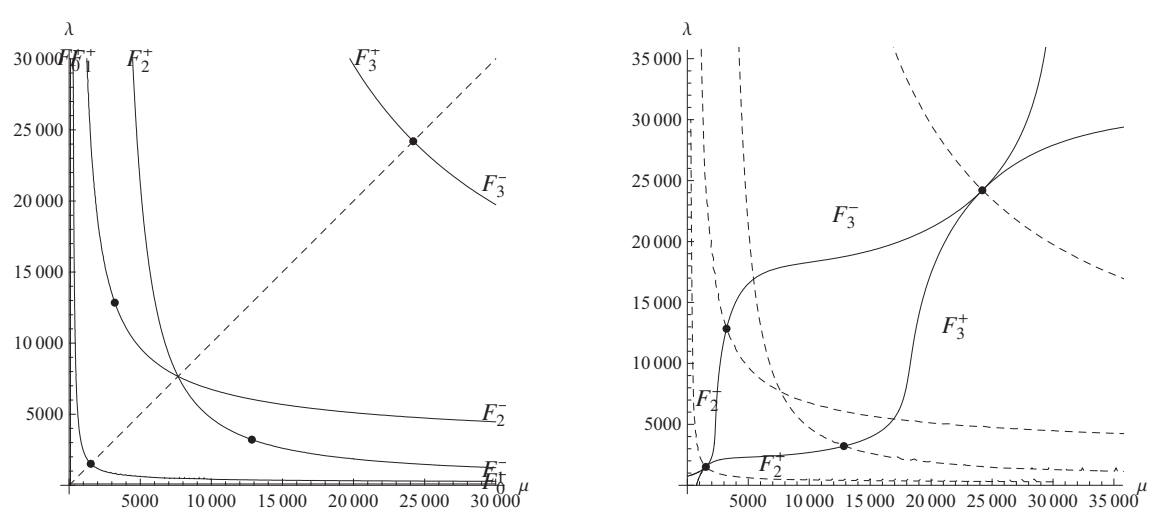

Figure 2. The spectrum for the problems (1.5), (2.1) and (1.5), (3.1).

\section{The Spectrum of the Problem with Zero Integral Con- dition}

Consider the equation (1.5) with the conditions

$$
x(0)=0, \quad \int_{0}^{1} x(s) d s=0, \quad\left|x^{\prime}(0)\right|=1 .
$$

Theorem 2. The Fučik spectrum for the problem (1.5), (3.1) consists of the branches given by

$$
\begin{aligned}
& F_{1}^{+}=\left\{(\mu, \lambda) \mid \frac{2 \pi}{4} \sqrt{\lambda}-\frac{\pi}{4} \sqrt{\mu}+\sqrt{\mu} \arctan c l(\sqrt[4]{\lambda / 2}-2 A \sqrt[4]{\lambda / \mu})=0,\right. \\
&\left.\mu>32 A^{4}, 2 A \sqrt[4]{2 / \mu}+2 A \sqrt[4]{2 / \lambda} \geq 1\right\} \\
& \cup\left\{(\mu, \lambda) \mid \mu=32(A+\sqrt{\pi / 4})^{4}, \lambda=0\right\}, \\
& F_{2 i}^{+}=\left\{(\mu, \lambda) \mid(2 i+1) \frac{\pi}{4} \sqrt{\lambda}-2 i \frac{\pi}{4} \sqrt{\mu}-\sqrt{\lambda} \arctan c l\left(\sqrt[4]{\frac{\mu}{2}}-2 A i \sqrt[4]{\frac{\mu}{\lambda}}\right.\right. \\
&\left.+2 A i)=0,2 A \sqrt[4]{\frac{2}{\mu}} i+2 A \sqrt[4]{\frac{2}{\lambda}} i<1,2 A \sqrt[4]{\frac{2}{\mu}} i+2 A \sqrt[4]{\frac{2}{\lambda}}(i+1) \geq 1\right\}, \\
& F_{2 i+1}^{+}=\left\{(\mu, \lambda) \mid(2 i+2) \frac{\pi}{4} \sqrt{\lambda}-(2 i+1) \frac{\pi}{4} \sqrt{\mu}-\sqrt{\mu} \arctan c l\left(\sqrt[4]{\frac{\lambda}{2}}\right.\right. \\
&\left.-2 A(i+1) \sqrt[4]{\frac{\lambda}{\mu}}+2 A(i+1)\right)=0,2 A \sqrt[4]{\frac{2}{\mu}}(i+1)+2 A \sqrt[4]{\frac{2}{\lambda}} i<1, \\
&2 A \sqrt[4]{2 / \mu}(i+1)+2 A \sqrt[4]{2 / \lambda}(i+1) \geq 1\}, \\
& F_{i}^{-}=\left\{(\mu, \lambda) \mid(\lambda, \mu) \in F_{i}^{+}\right\},
\end{aligned}
$$

where $\mathrm{cl}(t)$ is the lemniscatic cosine function, $A=\int_{0}^{1} \frac{d s}{\sqrt{1-s^{4}}}, i=1,2, \ldots$ 
Remark 1. The branches $F_{1}^{ \pm}$consist of two parts: the part in the first quadrant and the point on the axis. The point on the axis corresponds to the solution which consists of the lemniscatic sine function continued by straight line (not by lemniscatic sine function as in the first quadrant of $(\mu, \lambda)$ plane).

Some pairs of branches of the spectrum to the problem (1.5), (3.1) are presented in Fig. 2, the dashed curves in this figure are the branches of the spectrum for the problem (1.5), (2.1), which separate branches of the spectrum for the problem (1.5), (3.1).

\section{The Connection of the Spectra}

Consider the equation (1.5) with the conditions

$$
x(0)=0, \quad\left|x^{\prime}(0)\right|=1, \quad x(1)=\gamma \int_{0}^{1} x(s) d s, \quad \gamma \in \mathbb{R} .
$$

The expressions of the branches of the spectrum for the problem (1.5), (4.1) are given in the next theorem.

Theorem 3. The spectrum for the problem (1.5), (4.1) consists of the branches (if these branches exist for corresponding value of $\gamma$ ) given by

$$
\begin{aligned}
F_{0}^{+}= & \left\{(\mu, \lambda) \mid \sqrt[4]{\frac{2}{\mu}} \operatorname{sl} \sqrt[4]{\frac{\mu}{2}}=\gamma \sqrt{\frac{2}{\mu}}\left(\frac{\pi}{4}-\arctan \mathrm{cl} \sqrt[4]{\frac{\mu}{2}}\right), 0<\mu \leq 2(2 A)^{4}\right\}, \\
F_{1}^{+}= & \left\{(\mu, \lambda) \mid \frac{2 \pi \sqrt{\lambda}}{4} \gamma-\frac{\pi \sqrt{\mu}}{4} \gamma+\sqrt{\mu} \gamma \arctan \mathrm{cl}\left(\sqrt[4]{\frac{\lambda}{2}}-2 A i \sqrt[4]{\frac{\lambda}{\mu}}\right)\right. \\
+ & \left.\sqrt[4]{\frac{\mu^{2} \lambda}{2}} \mathrm{sl}\left(\sqrt[4]{\frac{\lambda}{2}}-2 A i \sqrt[4]{\frac{\lambda}{\mu}}\right)=0, \mu>32 A^{4}, 2 A \sqrt[4]{\frac{2}{\mu}}+2 A \sqrt[4]{\frac{2}{\lambda}} \geq 1\right\} \\
\cup & \left\{(\mu, \lambda) \mid 2 A \sqrt[4]{\frac{2}{\mu}}-1=\gamma\left(\frac{\pi}{2} \sqrt{\frac{2}{\mu}}+2 A \sqrt[4]{\frac{2}{\mu}}-2 A^{2} \sqrt{\frac{2}{\mu}}-\frac{1}{2}\right), \lambda=0\right\}, \\
F_{2 i}^{+}= & \left\{(\mu, \lambda) \mid \sqrt{\lambda} \gamma \operatorname{arctancl}\left(\sqrt[4]{\frac{\mu}{2}}-2 A i \sqrt[4]{\frac{\mu}{\lambda}}+2 A i\right)\right. \\
& +\sqrt[4]{\frac{\lambda^{2} \mu}{2}} \operatorname{sl}\left(\sqrt[4]{\frac{\mu}{2}}-2 A i \sqrt[4]{\frac{\mu}{\lambda}}+2 A i\right)-\frac{(2 i+1) \pi \sqrt{\lambda}}{4} \gamma+\frac{2 i \pi \sqrt{\mu}}{4} \gamma=0, \\
& \left.2 A \sqrt[4]{\frac{2}{\mu}} i+2 A \sqrt[4]{\frac{2}{\lambda}} i<1,2 A \sqrt[4]{\frac{2}{\mu}} i+2 A \sqrt[4]{\frac{2}{\lambda}}(i+1) \geq 1\right\} \\
F_{2 i+1}^{+}= & \left\{(\mu, \lambda) \mid \sqrt{\mu} \gamma \arctan \operatorname{cl}\left(\sqrt[4]{\frac{\lambda}{2}}-2 A(i+1) \sqrt[4]{\frac{\lambda}{\mu}}+2 A(i+1)\right)\right. \\
& +\sqrt[4]{\frac{\mu^{2} \lambda}{2}} \operatorname{sl}\left(\sqrt[4]{\frac{\lambda}{2}}-2 A(i+1) \sqrt[4]{\frac{\lambda}{\mu}}+2 A(i+1)\right)-\frac{(2 i+2) \pi \sqrt{\lambda}}{4} \gamma
\end{aligned}
$$




$$
\begin{aligned}
& +\frac{(2 i+1) \pi \sqrt{\mu}}{4} \gamma=0,2 A \sqrt[4]{\frac{2}{\mu}}(i+1)+2 A \sqrt[4]{\frac{2}{\lambda}} i<1,2 A \sqrt[4]{\frac{2}{\mu}}(i+1) \\
& \left.+2 A \sqrt[4]{\frac{2}{\lambda}}(i+1) \geq 1\right\}, \quad F_{i}^{-}=\left\{(\mu, \lambda) \mid(\lambda, \mu) \in F_{i}^{+}\right\}
\end{aligned}
$$

where $\operatorname{sl}(t), \operatorname{cl}(t)$ are the lemniscatic sine and cosine functions, $A=\int_{0}^{1} \frac{d s}{\sqrt{1-s^{4}}}$, $i=1,2, \ldots$.

Proof. The proof of this theorem is similar to the proof given in the works [10] or [12].

Some comments follow. First of all we obtain the expression for $F_{0}^{+}$. Let us suppose that the solution without zeroes in the interval $(0,1)$ exists and $x^{\prime}(0)=1$. In this case we obtain that the problem (1.5), (4.1) reduces to the eigenvalue problem

$$
-x^{\prime \prime}=\mu x^{3}, \quad x(0)=0, \quad x(1)=\gamma \int_{0}^{1} x(s) d s .
$$

The solution of the equation (4.2) which satisfies the first boundary condition is given by $x(t)=\sqrt[4]{2 / \mu} \mathrm{sl}(\sqrt[4]{\mu / 2} t)$. It must satisfy the integral condition from (4.2). From the work [10] we know that $\int_{0}^{t} \mathrm{sl} s d s=\frac{\pi}{4}-\arctan \mathrm{cl} t$. In view of $x(1)=\sqrt[4]{2 / \mu} \operatorname{sl}(\sqrt[4]{\mu / 2})$ and

$$
\int_{0}^{1} x(s) d s=\sqrt{2 / \mu}\left(\frac{\pi}{4}-\arctan \operatorname{cl}(\sqrt[4]{\mu / 2})\right)
$$

we obtain the expression for $F_{0}^{+}$.

The idea of the proof for other branches is similar. We consider the eigenvalue problems in the intervals between two consecutive zeroes of the solution and use the conditions of the solutions for these problems. For example, we will prove this theorem for $F_{1}^{+}$. Suppose that $(\mu, \lambda) \in F_{1}^{+}$and let $x(t)$ be the corresponding nontrivial solution of the problem (1.5), (4.1). The solution has only one zero in $(0,1)$ and $x^{\prime}(0)=1$. Let this zero be denoted by $\tau$. Consider a solution of the problem $(1.5),(4.1)$ in the interval $(0, \tau)$. We obtain that the problem (1.5), (4.1) in this interval reduces to the eigenvalue problem

$$
-x^{\prime \prime}=\mu x^{3}, \quad x(0)=x(\tau)=0 .
$$

Since $x(t)=\sqrt[4]{2 / \mu} \operatorname{sl}(\sqrt[4]{\mu / 2} t)$ in the interval $(0, \tau)$ and $x(\tau)=0$, we obtain that $\tau=2 A \sqrt[4]{2 / \mu}$. It follows that

$$
\int_{0}^{\tau} \sqrt[4]{2 / \mu} \operatorname{sl}(\sqrt[4]{\mu / 2} s) d s=\frac{\pi}{2} \sqrt{2 / \mu}
$$

Now consider a solution of the problem (1.5), (4.1) in the interval $(\tau, 1)$. We obtain the problem $-x^{\prime \prime}=\lambda x^{3}(\lambda>0)$ with boundary condition $x(\tau)=0$ in this interval. Since $x(t)=-\sqrt[4]{2 / \lambda} \operatorname{sl}(\sqrt[4]{\lambda / 2}(t-2 A \sqrt[4]{2 / \mu}))$ in the interval $(\tau, 1)$ we obtain that

$$
x(1)=-\sqrt[4]{2 / \lambda} \operatorname{sl}(\sqrt[4]{\lambda / 2}(1-2 A \sqrt[4]{2 / \mu}))
$$




$$
\begin{aligned}
& \int_{\tau}^{1}(-1) \sqrt[4]{2 / \lambda} \operatorname{sl}(\sqrt[4]{\lambda / 2}(s-2 A \sqrt[4]{2 / \mu})) d s \\
& \quad=\sqrt{2 / \lambda}\left(\arctan \mathrm{cl}(\sqrt[4]{\lambda / 2}(1-2 A \sqrt[4]{2 / \mu}))-\frac{\pi}{4}\right)
\end{aligned}
$$

Using (4.3), (4.4) and (4.5) in the condition $x(1)=\gamma \int_{0}^{1} x(s) d s$ we obtain the expression for $F_{1}^{+}$(for $\left.\gamma>0\right)$.

Now consider a solution of the problem (1.5), (4.1) which consists of the lemniscatic sine function continued by straight line or $x^{\prime \prime}=0$ in the interval $(\tau, 1)$. Since $x(t)=-t+2 A \sqrt[4]{2 / \mu}$ in the interval $(\tau, 1)$ we obtain the expression for finding the point on the axis.

Considering the solution of the problem (1.5), (4.1) it is easy to prove that

$$
0<2 A \sqrt[4]{2 / \mu}<1 \leq 2 A \sqrt[4]{2 / \mu}+2 A \sqrt[4]{2 / \lambda}
$$

The proof for other branches is analogous.

Some properties of the spectrum for the problem (1.5), (4.1) are given in the next lemmas.

Lemma 1. The problem (1.5), (4.1) connects both above considered problems. If $\gamma=0$ we obtain the problem (1.5), (2.1), but if $\gamma= \pm \infty$ we obtain the problem (1.5), (3.1).

Proof. The first part of Lemma is evident. Let us consider $\gamma= \pm \infty$. We rewrite the integral condition of the problem (1.5), (4.1) in the form (we can do it because $\gamma \neq 0$ )

$$
\frac{x(1)}{\gamma}=\int_{0}^{1} x(s) d s .
$$

The left side tends to zero and the integral tends to zero also. The proof of Lemma 1 follows.

Lemma 2. The point on the axis in the $F_{1}^{ \pm}$of the spectrum for the problem (1.5), (4.1) exists for $\gamma<0$ and $\gamma>2$.

Proof. Consider the equation

$$
2 A \sqrt[4]{\frac{2}{\mu}}-1=\gamma\left(\frac{\pi}{2} \sqrt{\frac{2}{\mu}}+2 A \sqrt[4]{\frac{2}{\mu}}-2 A^{2} \sqrt{\frac{2}{\mu}}-\frac{1}{2}\right) .
$$

The investigation of the (4.6) as a quadratic equation for $k=\sqrt[4]{2 / \mu}$ with parameter $\gamma$ shows that solutions of this equation exist for any real $\gamma$ values. These solutions satisfy the condition $\mu>32 A^{4}$ (which is necessary for one zero in the interval $(0,1))$ only for $\gamma<0$ and $\gamma>2$. This proves the lemma.

Lemma 3. The branches $F_{0}^{ \pm}$of the spectrum for the problem (1.5), (4.1) do not exist for $\gamma<0$. 
Proof. Consider the solution without zeroes in the interval $(0,1)$. It is clear that $\operatorname{sign} x^{\prime}(0)=\operatorname{sign} x(t)=\operatorname{sign} \int_{0}^{1} x(s) d s($ where $t \in(0,1))$. It follows that

$$
\operatorname{sign}\left(\gamma \int_{0}^{1} x(s) d s\right) \neq \operatorname{sign} x(t)
$$

for negative $\gamma$ values. Therefore the branches $F_{0}^{ \pm}$do not exist for $\gamma<0$.

Lemma 4. The spectrum of the problem (1.5), (4.1) is symmetric with respect the bisectrix of the first quadrant of the parameter $(\mu, \lambda)$ plane.

Proof. This lemma is a direct consequence of the proof of the Theorem 3.

Lemma 5. There are the points of $(\mu, \lambda)$-plane, which are fixed for any values of $\gamma$. For any values of $\gamma$, except $\gamma=0$, the odd-numbered and the evennumbered branches are separated by $\left(2(4 A i)^{4}, 2(4 A i)^{4}\right)$, but the even-numbered and the odd-numbered branches are separated by the points

$$
\begin{aligned}
& F_{2 i}^{+} \cap F_{2 i+1}^{+}=\left((\sqrt{i(i+1)}+i+1)^{4} 2(2 A)^{4} ;(\sqrt{i(i+1)}+i)^{4} 2(2 A)^{4}\right), \\
& F_{2 i}^{-} \cap F_{2 i+1}^{-}=\left((\sqrt{i(i+1)}+i)^{4} 2(2 A)^{4},(\sqrt{i(i+1)}+i+1)^{4} 2(2 A)^{4}\right),
\end{aligned}
$$

where $i=1,2, \ldots$

Proof. It is clear that the odd-numbered branches and the even-numbered ones intersect at the points in which the problem $(1.5),(4.1)$ reduces to the eigenvalue problem

$$
-x^{\prime \prime}=\mu x^{3}, \quad x(0)=0, \quad \int_{0}^{1} x(s) d s=0 .
$$

The eigenvalues of this problem are $\mu=2(4 A i)^{4}$. It follows the first assertion of lemma. The even-numbered branches $F_{2 i}^{+}$and the odd-numbered ones $F_{2 i+1}^{+}$ intersect at the points which satisfy the system of equations

$$
\left\{\begin{array}{l}
2 A \sqrt[4]{\frac{2}{\mu}}(i+1)+2 A \sqrt[4]{\frac{2}{\lambda}} i=1 \\
\frac{\pi}{2} \sqrt{\frac{2}{\mu}}(i+1)=\frac{\pi}{2} \sqrt{\frac{2}{\lambda}} i
\end{array}\right.
$$

In view of the second equation of the system (4.7) we obtain that $\lambda=\left(\frac{i}{i+1}\right)^{2} \mu$. The second assertion of Lemma 5 follows from the first equation of the system (4.7).

Several first branches of the spectrum to the problem (1.5), (4.1) for different $\gamma$ values are depicted in Fig. 3, the dashed curves is the spectrum for the problem $(1.5),(2.1)$, the other ones $-F_{i}^{+}$and $F_{i}^{-}$branches of the spectrum for the problem (1.5), (4.1). The spectrum of the problem (1.5), (4.1) for $\gamma=0$ and $\gamma= \pm \infty$ is shown in Fig. 2 as follows from Lemma 1. 

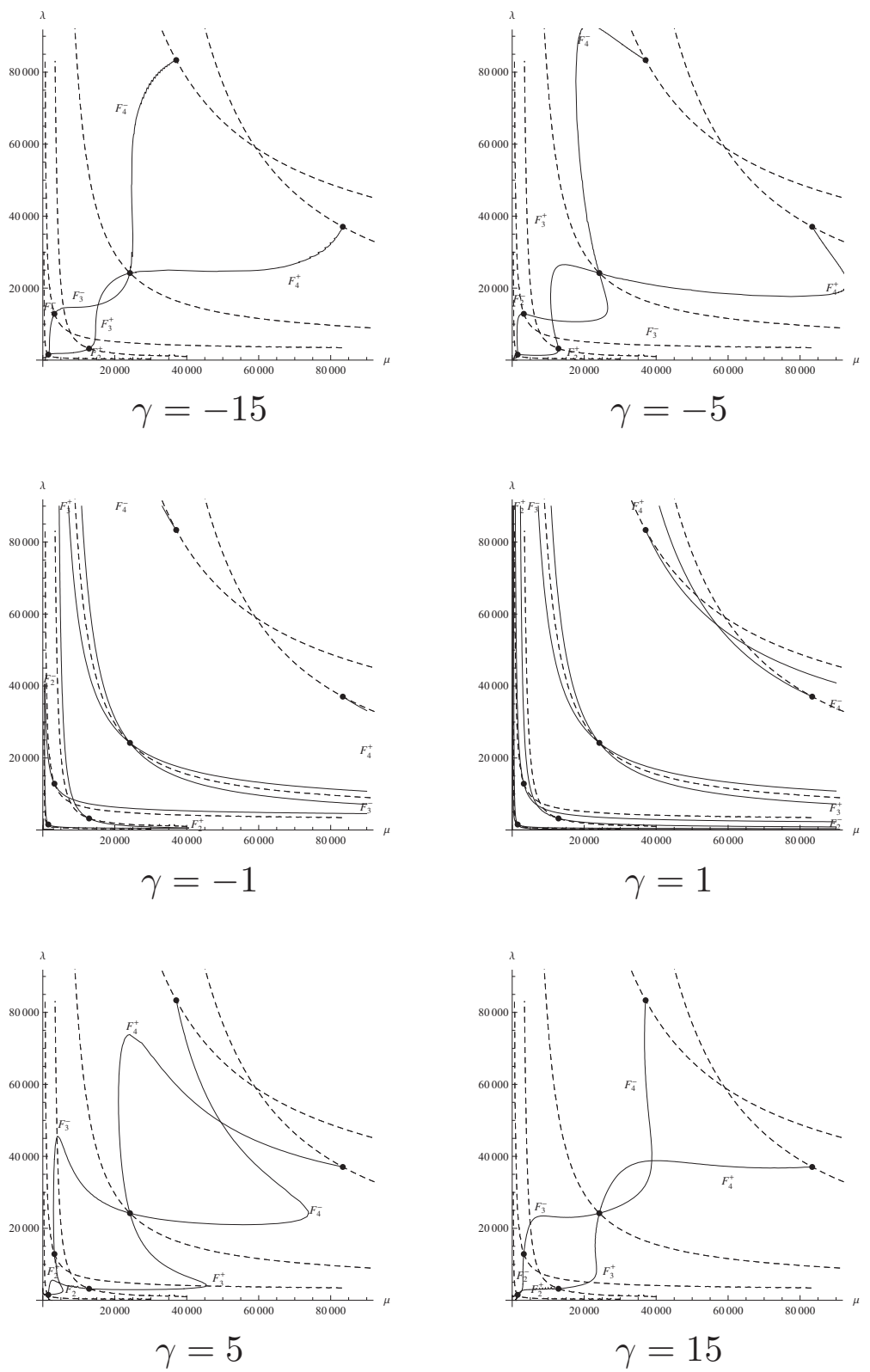

Figure 3. The spectrum for the problem (1.5), (4.1) for different $\gamma$ values.

\section{Conclusions}

The spectrum of the problem (1.5), (4.1) was investigated and the following properties of the spectrum were established:

- The spectrum for small $\gamma$ is similar to the Fučík type spectrum for Dirichlet problem, which was considered in [8].

- The spectrum for large $\gamma$ is similar to the Fučík type spectrum for the integral problem, the analog of this problem was considered in [10].

- Passage from $\gamma=0$ to $\gamma= \pm \infty$ is different for $\gamma>0$ and $\gamma<0$ (for example, the asymptotic of the branches is different). 
- Some branches of the spectrum $\left(F_{0}^{+}\right.$and $\left.F_{0}^{-}\right)$do not exist for some values of $\gamma$.

- The spectrum can be visualized by using analytical representation of solutions via the Jacobi elliptic functions. The respective diagrams were constructed in the article.

\section{References}

[1] R. Čiupaila, Ž. Jesevičiūtè and M. Sapagovas. On the eigenvalue problem for one-dimensional differential operator with nonlocal integral condition. Nonlinear Anal. Model. Control, 9(2):109-116, 2004.

[2] A. Gritsans and F. Sadyrbaev. Remarks on lemniscatic functions. Acta Math. Latviensis, 688:39-50, 2005.

[3] A. Gritsans and F. Sadyrbaev. On nonlinear fučík type spectra. Math. Model. Anal., 13(2):203-210, 2008. Doi:10.3846/1392-6292.2008.13.203-210.

[4] A. Kufner and S. Fučík. Nonlinear Differential Equations. Nauka, Moscow, 1988.

[5] S. Pečiulytè, O. Štikonienè and A. Štikonas. Sturm-Liouville problem for stationary differential operator with nonlocal integral boundary condition. Math. Model. Anal., 10(4):377-392, 2005. Doi:10.1080/13926292.2005.9637295.

[6] S. Pečiulytè, O. Štikonienè and A. Štikonas. Investigation of negative critical points of the characteristic function for problems with nonlocal boundary conditions. Nonlinear Anal. Model. Control, 13(4):467-490, 2008.

[7] S. Roman and A. Štikonas. Green's functions for stationary problems with nonlocal boundary conditions. Lith. Math. J., 49(2):190-202, 2009.

Doi:10.1007/s10986-009-9041-0.

[8] F. Sadyrbaev and A. Gritsans. Nonlinear spectra for parameter dependent ordinary differential equations. Nonlinear Anal. Model. Control, 12(2):253-267, 2007.

[9] N. Sergejeva. On the unusual Fučík spectrum. In B. Belinsky, K. Lan, X. Lu, M. Miranville and R. Shivaji(Eds.), Proc. for the 6th AIMS International Conference on DCDE, France, 2006, pp. 920-926. AIMS, 2007.

[10] N. Sergejeva. On nonlinear spectra for some nonlocal boundary value problems. Math. Model. Anal., 13(1):87-98, 2008. Doi:10.3846/1392-6292.2008.13.87-97.

[11] N. Sergejeva. On nonlinear spectra for some nonlocal boundary value problems. Electron. J. Qual. Theory Differ. Equ., 19:1-12, 2008.

[12] N. Sergejeva. On some problems with nonlocal integral condition. Math. Model. Anal., 15(1):113-126, 2010. Doi:10.3846/1392-6292.2010.15.113-126.

[13] A. Śtikonas and S. Roman. Stationary problems with two additional conditions and formulae for Green's functions. Numer. Funct. Anal. Optim., 30(9):11251144, 2009. Doi:10.1080/01630560903420932.

[14] E.T. Whittaker and G.N. Watson. A Course of Modern Analysis, Part II. Cambridge Univ. Press, 1927. 